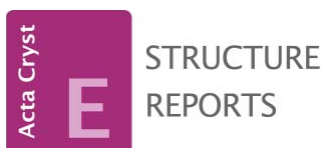

ISSN 1600-5368

Received 11 July 2014

Accepted 2 October 2014

Edited by M. Weil, Vienna University of Technology, Austria

Keywords: crystal structure; mild hydrothermal synthesis; iron phosphite; IR spectroscopy

CCDC reference: 1027279

Supporting information: this article has supporting information at journals.iucr.org/e

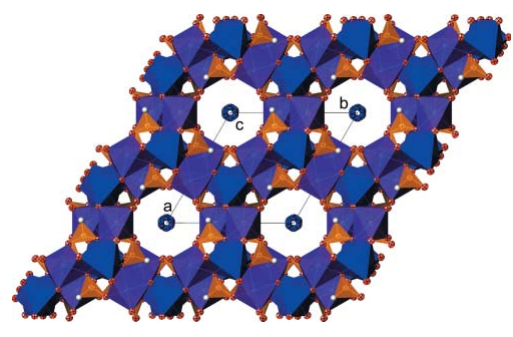

OPEN $\odot$ ACCESS

\section{Crystal structure of $\left(\mathrm{NH}_{4}\right)_{2}\left[\mathrm{Fe}_{5}{ }_{5}\left(\mathrm{HPO}_{3}\right)_{6}\right]$, a new open-framework phosphite}

\author{
Teresa Berrocal, ${ }^{a}$ Jose Luis Mesa, ${ }^{a}$ Edurne Larrea $^{\mathrm{b} *}$ and Juan Manuel Arrieta ${ }^{\mathrm{a}}$ \\ aDpto. de Química Inorgánica, Facultad de Ciencia y Tecnología, Universidad del País Vasco, UPV/EHU, 48080 Leioa, \\ Spain, and ${ }^{\mathbf{b}}$ Dpto. Mineralogía y Petrología, Facultad Ciencia y Tecnología, Universidad del País Vasco, UPV/EHU, \\ 48080 Leioa, Spain. *Correspondence e-mail: edurne.serrano@ehu.es
}

Diammonium hexaphosphitopentaferrate(II), $\left(\mathrm{NH}_{4}\right)_{2}\left[\mathrm{Fe}_{5}\left(\mathrm{HPO}_{3}\right)_{6}\right]$, was synthesized under mild hydrothermal conditions and autogeneous pressure, yielding twinned crystals. The crystal structure exhibits an $\left[\mathrm{Fe}_{5}{ }_{5}\left(\mathrm{HPO}_{3}\right)_{6}\right]^{2-}$ open framework with $\mathrm{NH}_{4}{ }^{+}$groups as counter-cations. The anionic skeleton is based on (001) sheets of $\left[\mathrm{FeO}_{6}\right]$ octahedra (one with point-group symmetry 3 .. and one with .2.) linked along [001] through $\left[\mathrm{HPO}_{3}\right]^{2-}$ oxoanions. Each sheet is constructed from 12-membered rings of edge-sharing $\left[\mathrm{FeO}_{6}\right]$ octahedra, giving rise to channels with a radius of $c a 3.1 \AA$ in which the disordered $\mathrm{NH}_{4}{ }^{+}$cations are located. The IR spectrum shows vibrational bands typical for phosphite and ammonium groups.

\section{Chemical context}

Research in the area of solids exhibiting open-framework structures continues to be exciting because of their numerous potential applications (Barrer, 1982; Hagrman et al., 1999). Prior to the early 1980s when nanoporous aluminium phosphates were first reported by Flanigen and co-workers, aluminosilicate-based zeolites (Wilson et al., 1982) and closely related systems represented the predominant class of materials with open-framework structures. In the meantime, a plethora of activities and efforts have been undertaken for the synthesis of numerous other compounds with open-framework structures of different dimensionalities (Yu \& Xu, 2006).

Recently a new ammonium iron phosphite, $\left(\mathrm{NH}_{4}\right)$ $\left[\mathrm{Fe}\left(\mathrm{HPO}_{3}\right)_{2}\right]$, has been reported (Hamchaoui et al., 2013) that consists of $\left[\mathrm{Fe}^{\mathrm{III}}\left(\mathrm{HPO}_{3}\right)_{2}\right]^{-}$layers formed by $\left[\mathrm{FeO}_{6}\right]$ octahedra interconnected by $\left[\mathrm{HPO}_{3}\right]^{2-}$ oxoanions. The ammonium counter-cations are located in the interlayer space. Here we report on synthesis and the crystal structure of another ammonium iron phosphite, $\left(\mathrm{NH}_{4}\right)_{2}\left[\mathrm{Fe}_{5}\left(\mathrm{HPO}_{3}\right)_{6}\right]$, in which iron exhibits oxidation state + II.

\section{Structural commentary}

Tha asymmetric unit of $\left(\mathrm{NH}_{4}\right)_{2}\left[\mathrm{Fe}_{5}\left(\mathrm{HPO}_{3}\right)_{6}\right]$ is displayed in Fig. 1. The crystal structure of the title compound contains $\left[\mathrm{FeO}_{6}\right]$ octahedra linked via edge-sharing into sheets parallel to (001). These sheets consist of 12 -membered rings whereby each ring is formed by six $\left[\mathrm{Fe}(1) \mathrm{O}_{6}\right]$ octahedra and six $\left[\mathrm{Fe}(2) \mathrm{O}_{6}\right]$ octahedra. The iron(II) ions occupy two different special positions ( $6 f$ and $4 d$ ) with site symmetries of .2. and 3.., respectively. In one of the $\mathrm{FeO}_{6}$ octahedra $(\mathrm{Fe} 1)$, the $\mathrm{Fe}-\mathrm{O}$ bond lengths range from 2.030 (2) to 2.217 (3) $\AA$ while in the $\left[\mathrm{Fe}(2) \mathrm{O}_{6}\right]$ octahedron a more uniform bond-length 


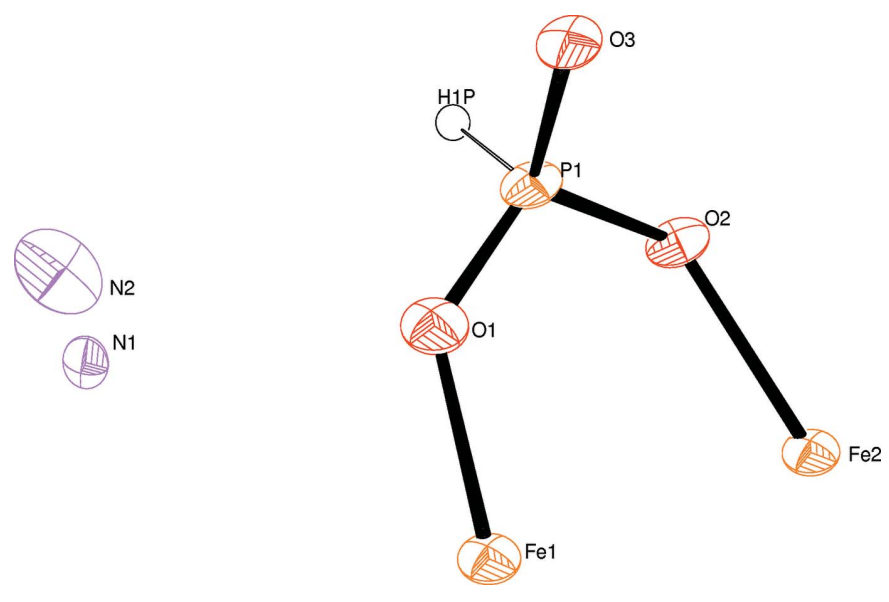

Figure 1

The asymmetric unit of $\left(\mathrm{NH}_{4}\right)_{2}\left[\mathrm{Fe}^{\mathrm{II}}{ }_{5}\left(\mathrm{HPO}_{3}\right)_{6}\right]$, with displacement parameters drawn at the $50 \%$ probability level.

distribution from 2.138 (3) to 2.140 (3) $\AA$ is observed. The bond angles of the two $\left[\mathrm{FeO}_{6}\right]$ octahedra range between $76.48(10)$ and 103.18 (9) ${ }^{\circ}$ for the cis- and between 163.65 (12) and $178.24(17)^{\circ}$ for the trans-angles.

The iron oxide sheets are linked through phosphite groups in which six anions share the most interior oxygen atoms of each ring (Fig. 2), forming 12-membered channels along [001] with a radius of about $3.1 \AA$. The phosphorus(III) atom of the complex oxoanion is located on a general position of this space group. The $\mathrm{P}-\mathrm{O}$ bond lengths of the anion range from 1.514 (3) to 1.538 (3) $\AA$, and the $\mathrm{P}-\mathrm{H}$ distance is 1.28 (5) $\AA$, with $\mathrm{O}-\mathrm{P}-\mathrm{O}$ bond angles from 110.28 (17) to $114.29(17)^{\circ}$.

\section{Supramolecular features}

The ammonium cations are located in the 12-membered channels of the framework structure. Although no hydrogen atoms of the cations could be located due to the positional disorder, $\mathrm{N} \cdots \mathrm{O}$ contacts of $2.67(6), 2.85(7), 2.87(8)$ and

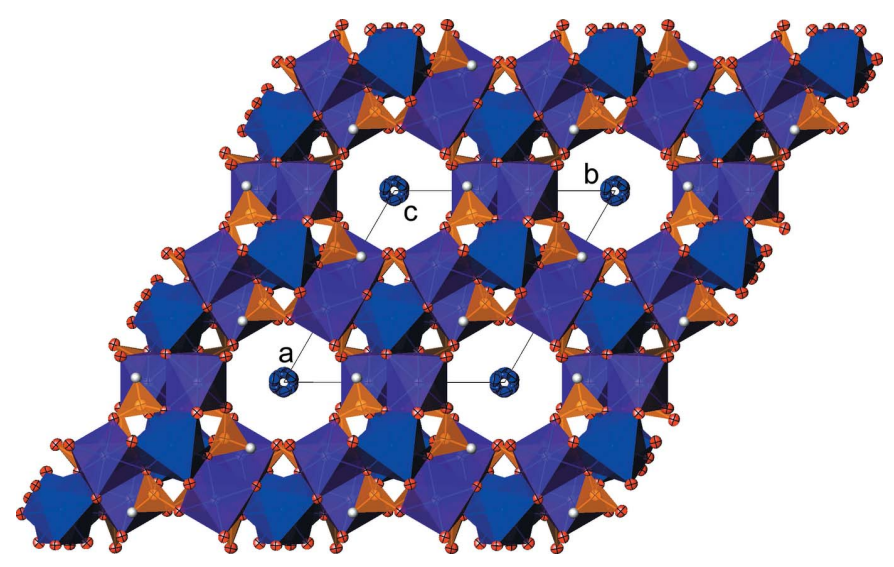

Figure 2

The crystal structure of $\left(\mathrm{NH}_{4}\right)_{2}\left[\mathrm{Fe}^{\mathrm{II}}{ }_{5}\left(\mathrm{HPO}_{3}\right)_{6}\right]$ in polyhedral representation, in a projection along [001]. Displacement parameters are drawn at the $50 \%$ probability level.

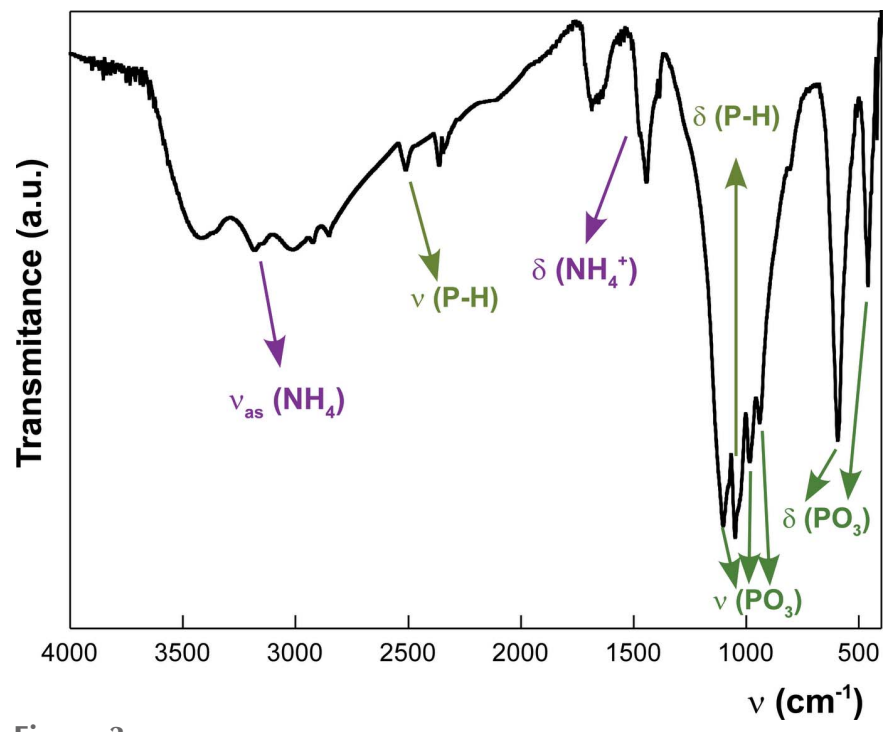

Figure 3

The IR spectrum of $\left(\mathrm{NH}_{4}\right)_{2}\left[\mathrm{Fe}_{5}^{\mathrm{II}}\left(\mathrm{HPO}_{3}\right)_{6}\right]$, with partial band assignments.

2.98 (6) $\AA$ between the cations and the $\mathrm{O}$ atoms of the anions suggest hydrogen-bonding interactions of medium strength. The $\mathrm{H}$ atom of the $\left[\mathrm{HPO}_{3}\right]^{2-}$ anion shows a distance of $2.51(3) \AA$ to atom $\mathrm{O} 1\left[\mathrm{P}-\mathrm{H} \cdots \mathrm{O}\right.$ angle $\left.116.8(14)^{\circ}\right]$ and seems not to be part of relevant hydrogen-bonding interactions.

\section{Synthesis and characterization}

$\left(\mathrm{NH}_{4}\right)_{2}\left[\mathrm{Fe}_{5}^{\mathrm{II}}\left(\mathrm{HPO}_{3}\right)_{6}\right]$ was synthesized under mild hydrothermal conditions and autogeneous pressure (10-20 bar at $343 \mathrm{~K}$ ). The reaction mixture was prepared from $30 \mathrm{ml}$ water, $2 \mathrm{ml}$ of phosphorous acid, $0.17 \mathrm{mmol}$ of $\mathrm{NH}_{4} \mathrm{OH}$ and $0.37 \mathrm{mmol}$ of $\mathrm{FeCl}_{3}$. The mixture had a $\mathrm{pH}$ value of $\simeq 6.0$. The reaction mixture was sealed in a polytetrafluoroethylene (PTFE)-lined steel pressure vessel, which was maintained at $343 \mathrm{~K}$ for five days. This procedure apparently caused reduction of iron(III) to iron(II) and led to the formation of single crystals of the title compound with a dark-green colour. All crystals appeared to be twinned. The presence of ammonium cations in the title compound was confirmed by infra-red spectroscopy, showing bands at 3190 and $1450 \mathrm{~cm}^{-1}$. Characteristic bands of the phosphite $\mathrm{P}-\mathrm{H}$ group were also observed at 2510 and $1050 \mathrm{~cm}^{-1}$ (Nakamoto, 1997).

\section{Refinement}

Crystal data, data collection and structure refinement details are summarized in Table 1. The title crystal was confirmed to be twinned by merohedry using the TwinRotMap option in PLATON (Spek, 2009). The twin element is a $180^{\circ}$-rotation around the $<1 \overline{1} 0>$ direction, or any other equivalent representations of the coset decomposition of the $6 / \mathrm{mmm}$ holohedry under crystal class $\overline{3} m 1$. The twin law $(0 \overline{1} 0 / \overline{1} 00 / 00 \overline{1})$ was used during the refinements, and the twin volume of the second component refined to a value of 0.079 (1)\%. 
Table 1

Experimental details.

\begin{tabular}{|c|c|}
\hline \multicolumn{2}{|l|}{ Crystal data } \\
\hline Chemical formula & $\left(\mathrm{NH}_{4}\right)_{2}\left[\mathrm{Fe}_{5}\left(\mathrm{HPO}_{3}\right)_{6}\right]$ \\
\hline$M_{\mathrm{r}}$ & 795.20 \\
\hline Crystal system, space group & Trigonal, $P \overline{3} c 1$ \\
\hline Temperature $(\mathrm{K})$ & 100 \\
\hline$a, c(\AA)$ & $10.3862(15), 9.2089(14)$ \\
\hline$V\left(\AA^{3}\right)$ & $860.3(3)$ \\
\hline$Z$ & 2 \\
\hline Radiation type & Мо $K \alpha$ \\
\hline$\mu\left(\mathrm{mm}^{-1}\right)$ & 4.78 \\
\hline Crystal size (mm) & $0.18 \times 0.05 \times 0.02$ \\
\hline \multicolumn{2}{|l|}{ Data collection } \\
\hline Diffractometer & $\begin{array}{l}\text { Agilent SuperNova (single source } \\
\text { at offset) }\end{array}$ \\
\hline Absorption correction & $\begin{array}{l}\text { Gaussian (CrysAlis PRO; Agilent, } \\
\text { 2014) }\end{array}$ \\
\hline$T_{\min }, T_{\max }$ & $0.566,0.893$ \\
\hline $\begin{array}{l}\text { No. of measured, independent and } \\
\text { observed }[I>2 \sigma(I)] \text { reflections }\end{array}$ & $6343,659,618$ \\
\hline$R_{\text {int }}$ & 0.073 \\
\hline$(\sin \theta / \lambda)_{\max }\left(\AA^{-1}\right)$ & 0.649 \\
\hline \multicolumn{2}{|l|}{ Refinement } \\
\hline$R\left[F^{2}>2 \sigma\left(F^{2}\right)\right], w R\left(F^{2}\right), S$ & $0.033,0.065,1.17$ \\
\hline No. of reflections & 659 \\
\hline No. of parameters & 68 \\
\hline No. of restraints & 15 \\
\hline $\mathrm{H}$-atom treatment & Only H-atom coordinates refined \\
\hline$\Delta \rho_{\max }, \Delta \rho_{\min }\left(\mathrm{e} \AA^{-3}\right)$ & $0.53,-0.76$ \\
\hline
\end{tabular}

Computer programs: CrysAlis PRO (Agilent, 2014), SUPERFLIP (Palatinus \& Chapuis, 2007), SHELXL2014 (Sheldrick, 2008), DIAMOND (Brandenburg, 2010) and OLEX2 (Dolomanov et al., 2009).

The hydrogen atom of the phosphite group was located in a difference map and restrained to be equidistant to the three oxygen atoms of the group, and a fixed isotropic displacement parameter with a value equal to $1.2 U_{\text {eq }}$ of the parent $\mathrm{P}$ atom was assigned.

The ammonium cation is equally disordered around a threefold rotation axis along $(00 z)$ and was refined with two positions, N1 and N2. The occupancy factors of N1 and N2 were initially freely refined, but since they refined close to the expected value of $1 / 6$, this value was fixed during the last cycles. Because the ellipsoids of these atoms were very elongated, ISOR commands of SHELXL2014 (Sheldrick, 2008) were used to achieve more regular displacements. This command restrains the $U_{\mathrm{ij}}$ components of anisotropically refined atoms to behave approximately isotropically within a standard uncertainty. $\mathrm{H}$ atoms belonging to the disordered ammonium atoms were not considered in the final model.

\section{Acknowledgements}

We gratefully acknowledge financial support of this work by te Gobierno Vasco (SAI12/82). The authors also thank the technicians of SGIkers, Dr J. Sangüesa, Dr Leire San Felices and Dr A. Larrañaga, financed by the National Program for the Promotion of Human Resources within the National Plan of Scientific Research, Development and Innovation, and the Ministerio de Ciencia y Tecnologia and Fondo Social Europeo (FSE), for the X-ray diffraction measurements. ESL thanks the Basque Government for her postdoctoral contract.

\section{References}

Agilent (2014). CrysAlis PRO. Agilent Technologies UK Ltd, Yarnton, England

Barrer, R. M. (1982). In Hydrothermal Chemistry of Zeolites. London: Academic Press.

Brandenburg, K. (2010). DIAMOND. Crystal Impact GbR, Bonn, Germany

Dolomanov, O. V., Bourhis, L. J., Gildea, R. J., Howard, J. A. K. \& Puschmann, H. (2009). J. Appl. Cryst. 42, 339-341.

Hagrman, P. J., Hagrman, D. \& Zubieta, J. (1999). Angew. Chem. Int. $E d$. 38, 2638-2684.

Hamchaoui, F., Alonzo, V., Venegas-Yazigi, D., Rebbah, H. \& Le Fur, E. (2013). J. Solid State Chem. 198, 295-302.

Nakamoto, K. (1997). In Infrared and Raman Spectroscopy of Inorganic and Coordination Compounds. New York: John Wiley \& Sons.

Palatinus, L. \& Chapuis, G. (2007). J. Appl. Cryst. 40, 786-790.

Sheldrick, G. M. (2008). Acta Cryst. A64, 112-122.

Spek, A. L. (2009). Acta Cryst. D65, 148-155.

Wilson, S. T., Lok, B. M., Messina, C. A., Cannan, T. R. \& Flanigen,

E. D. (1982). J. Am. Chem. Soc. 104, 1146-1147.

Yu, J. \& Xu, R. (2006). Chem. Soc. Rev. 35, 593-604. 


\section{supporting information}

Acta Cryst. (2014). E70, 309-311 [doi:10.1107/S1600536814021783]

\section{Crystal structure of $\left(\mathrm{NH}_{4}\right)_{2}\left[\mathrm{Fe}_{5}{ }_{5}\left(\mathrm{HPO}_{3}\right)_{6}\right]$, a new open-framework phosphite}

\section{Teresa Berrocal, Jose Luis Mesa, Edurne Larrea and Juan Manuel Arrieta}

\section{Computing details}

Data collection: CrysAlis PRO (Agilent, 2014); cell refinement: CrysAlis PRO (Agilent, 2014); data reduction: CrysAlis PRO (Agilent, 2014); program(s) used to solve structure: SUPERFLIP (Palatinus \& Chapuis, 2007); program(s) used to refine structure: SHELXL2014 (Sheldrick, 2008); molecular graphics: DIAMOND (Brandenburg, 2010); software used to prepare material for publication: OLEX2 (Dolomanov et al., 2009).

\section{Diammonium hexaphosphitopentaferrate(II)}

Crystal data

$\left(\mathrm{NH}_{4}\right)_{2}\left[\mathrm{Fe}_{5}\left(\mathrm{HPO}_{3}\right)_{6}\right]$

$M_{r}=795.20$

Trigonal, $P \overline{3} c 1$

$a=10.3862(15) \AA$

$c=9.2089(14) \AA$

$V=860.3(3) \AA^{3}$

$Z=2$

$F(000)=784$

Data collection

Agilent SuperNova (single source at offset) diffractometer

Radiation source: SuperNova (Mo) X-ray

Source

Mirror monochromator

Detector resolution: 16.2439 pixels $\mathrm{mm}^{-1}$

$\omega$ scans

Absorption correction: gaussian

(CrysAlis PRO; Agilent, 2014)

Refinement

Refinement on $F^{2}$

Least-squares matrix: full

$R\left[F^{2}>2 \sigma\left(F^{2}\right)\right]=0.033$

$w R\left(F^{2}\right)=0.065$

$S=1.17$

659 reflections

68 parameters

15 restraints

Primary atom site location: structure-invariant direct methods
$D_{\mathrm{x}}=3.070 \mathrm{Mg} \mathrm{m}^{-3}$

Mo $K \alpha$ radiation, $\lambda=0.71073 \AA$

Cell parameters from 2414 reflections

$\theta=2.3-28.3^{\circ}$

$\mu=4.78 \mathrm{~mm}^{-1}$

$T=100 \mathrm{~K}$

Acicular, dark green

$0.18 \times 0.05 \times 0.02 \mathrm{~mm}$

$T_{\min }=0.566, T_{\max }=0.893$

6343 measured reflections

659 independent reflections

618 reflections with $I>2 \sigma(I)$

$R_{\text {int }}=0.073$

$\theta_{\max }=27.5^{\circ}, \theta_{\min }=2.2^{\circ}$

$h=-13 \rightarrow 11$

$k=-10 \rightarrow 13$

$l=-11 \rightarrow 11$

Secondary atom site location: difference Fourier map

Hydrogen site location: difference Fourier map

Only H-atom coordinates refined

$w=1 /\left[\sigma^{2}\left(F_{\mathrm{o}}^{2}\right)+(0.0126 P)^{2}+2.2823 P\right]$

where $P=\left(F_{\mathrm{o}}{ }^{2}+2 F_{\mathrm{c}}{ }^{2}\right) / 3$

$(\Delta / \sigma)_{\max }<0.001$

$\Delta \rho_{\max }=0.53 \mathrm{e} \AA^{-3}$

$\Delta \rho_{\min }=-0.76$ e $\AA^{-3}$ 


\section{Special details}

Geometry. All e.s.d.'s (except the e.s.d. in the dihedral angle between two 1.s. planes) are estimated using the full covariance matrix. The cell e.s.d.'s are taken into account individually in the estimation of e.s.d.'s in distances, angles and torsion angles; correlations between e.s.d.'s in cell parameters are only used when they are defined by crystal symmetry. An approximate (isotropic) treatment of cell e.s.d.'s is used for estimating e.s.d.'s involving 1.s. planes.

Refinement. Refinement of $F^{2}$ against ALL reflections. The weighted $R$-factor $w R$ and goodness of fit $S$ are based on $F^{2}$, conventional $R$-factors $R$ are based on $F$, with $F$ set to zero for negative $F^{2}$. The threshold expression of $F^{2}>2 \sigma\left(F^{2}\right)$ is used only for calculating $R$-factors(gt) etc. and is not relevant to the choice of reflections for refinement. $R$-factors based on $F^{2}$ are statistically about twice as large as those based on $F$, and $R$ - factors based on ALL data will be even larger.

Fractional atomic coordinates and isotropic or equivalent isotropic displacement parameters $\left(\AA^{2}\right)$

\begin{tabular}{llllll}
\hline & $x$ & $y$ & $z$ & $U_{\text {iso }} * / U_{\text {eq }}$ & Occ. $(<1)$ \\
\hline Fe1 & $0.61330(9)$ & 0.0000 & 0.2500 & $0.0209(2)$ & \\
Fe2 & 0.6667 & 0.3333 & $0.33453(10)$ & $0.0202(3)$ & \\
P1 & $0.88002(13)$ & $0.29352(13)$ & $0.09044(10)$ & $0.0224(3)$ & \\
H1P & $1.020(5)$ & $0.343(3)$ & $0.091(2)$ & $0.027^{*}$ & \\
O2 & $0.8512(3)$ & $0.3935(3)$ & $0.1927(3)$ & $0.0234(7)$ & \\
O3 & $0.8404(3)$ & $0.3102(4)$ & $-0.0665(3)$ & $0.0243(7)$ & \\
O1 & $0.8063(4)$ & $0.1345(4)$ & $0.1440(3)$ & $0.0284(8)$ & \multirow{2}{*}{0.1667} \\
N2 & $0.970(5)$ & $-0.047(3)$ & $0.059(2)$ & $0.045(9)$ & 0.1667 \\
N1 & $1.024(5)$ & $-0.015(6)$ & $0.2033(17)$ & $0.020(7)$ & \\
\hline
\end{tabular}

Atomic displacement parameters $\left(\AA^{2}\right)$

\begin{tabular}{lllllll}
\hline & $U^{11}$ & $U^{22}$ & $U^{33}$ & $U^{12}$ & $U^{13}$ & $U^{23}$ \\
\hline Fe1 & $0.0286(4)$ & $0.0250(5)$ & $0.0080(4)$ & $0.0125(2)$ & $0.00013(16)$ & $0.0003(3)$ \\
Fe2 & $0.0264(4)$ & $0.0264(4)$ & $0.0077(4)$ & $0.01319(19)$ & 0.000 & 0.000 \\
P1 & $0.0278(6)$ & $0.0323(7)$ & $0.0066(4)$ & $0.0148(5)$ & $-0.0011(4)$ & $-0.0018(4)$ \\
O2 & $0.0265(17)$ & $0.0314(17)$ & $0.0088(12)$ & $0.0119(14)$ & $-0.0013(11)$ & $-0.0049(12)$ \\
O3 & $0.0330(18)$ & $0.0366(18)$ & $0.0062(11)$ & $0.0195(15)$ & $-0.0043(11)$ & $-0.0037(12)$ \\
O1 & $0.041(2)$ & $0.0311(18)$ & $0.0123(13)$ & $0.0176(16)$ & $-0.0007(13)$ & $-0.0011(13)$ \\
N2 & $0.036(17)$ & $0.040(17)$ & $0.051(11)$ & $0.014(13)$ & $-0.015(15)$ & $0.004(10)$ \\
N1 & $0.012(17)$ & $0.011(15)$ & $0.030(8)$ & $0.002(7)$ & $0.002(9)$ & $-0.002(10)$ \\
\hline
\end{tabular}

Geometric parameters $\left(\AA,{ }^{\circ}\right)$

\begin{tabular}{llll}
\hline $\mathrm{Fe} 1-\mathrm{O} 2^{\mathrm{i}}$ & $2.086(3)$ & $\mathrm{Fe} 2-\mathrm{O} 3^{\text {vii }}$ & $2.140(3)$ \\
$\mathrm{Fe} 1-\mathrm{O}^{\text {ii }}$ & $2.086(3)$ & $\mathrm{Fe} 2-\mathrm{O} 3^{\text {vii }}$ & $2.140(3)$ \\
$\mathrm{Fe} 1-\mathrm{O}^{\text {iii }}$ & $2.217(3)$ & $\mathrm{P} 1-\mathrm{O} 2$ & $1.538(3)$ \\
$\mathrm{Fe} 1-\mathrm{O} 3^{\text {iv }}$ & $2.217(3)$ & $\mathrm{P} 1-\mathrm{O} 3$ & $1.536(3)$ \\
$\mathrm{Fe} 1-\mathrm{O} 1$ & $2.030(3)$ & $\mathrm{P} 1-\mathrm{O} 1$ & $1.514(3)$ \\
$\mathrm{Fe} 1-\mathrm{O} 1^{\mathrm{v}}$ & $2.030(3)$ & $\mathrm{O} 2-\mathrm{Fe}^{\mathrm{vi}}$ & $2.086(3)$ \\
$\mathrm{Fe} 2-\mathrm{O} 2$ & $2.138(3)$ & $\mathrm{O} 3-\mathrm{Fe}^{\mathrm{ix}}$ & $2.217(3)$ \\
$\mathrm{Fe} 2-\mathrm{O} 2^{\mathrm{ii}}$ & $2.138(3)$ & $\mathrm{O} 3-\mathrm{Fe} 2^{\mathrm{x}}$ & $2.140(3)$ \\
$\mathrm{Fe} 2-\mathrm{O}^{\mathrm{vi}}$ & $2.138(3)$ & $\mathrm{P} 1-\mathrm{H} 1 \mathrm{P}$ & $1.28(5)$ \\
$\mathrm{Fe} 2-\mathrm{O}^{\mathrm{iii}}$ & $2.140(3)$ & &
\end{tabular}




\begin{tabular}{|c|c|c|c|}
\hline $\mathrm{O} 2^{\mathrm{i}}-\mathrm{Fe} 1-\mathrm{O} 2^{\mathrm{ii}}$ & $87.23(17)$ & $\mathrm{O} 2-\mathrm{Fe} 2-\mathrm{O}^{\text {vii }}$ & $163.65(11)$ \\
\hline $\mathrm{O} 2^{\mathrm{ii}-}-\mathrm{Fe} 1-\mathrm{O}^{\mathrm{iv}}$ & $102.21(11)$ & $\mathrm{O} 2-\mathrm{Fe} 2-\mathrm{O} 3^{\text {viii }}$ & $77.08(11)$ \\
\hline $\mathrm{O} 2^{\mathrm{ii}-}-\mathrm{Fe} 1-\mathrm{O}^{\mathrm{iii}}$ & $76.48(10)$ & 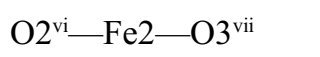 & $77.08(11)$ \\
\hline $\mathrm{O} 2^{\mathrm{i}}-\mathrm{Fe} 1-\mathrm{O}^{3 \mathrm{iv}}$ & $76.48(10)$ & $\mathrm{O} 2^{\mathrm{ii}}-\mathrm{Fe} 2-\mathrm{O}^{\text {viii }}$ & $163.65(11)$ \\
\hline $\mathrm{O} 2^{\mathrm{i}}-\mathrm{Fe} 1-\mathrm{O} 3^{\mathrm{iii}}$ & $102.21(11)$ & 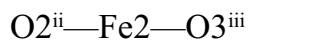 & $77.08(11)$ \\
\hline $\mathrm{O}^{\mathrm{iv}}-\mathrm{Fe} 1-\mathrm{O}^{3 \mathrm{ii}}$ & $178.24(17)$ & 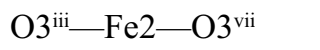 & $103.18(9)$ \\
\hline $\mathrm{O} 1^{\mathrm{v}}-\mathrm{Fe} 1-\mathrm{O} 2^{\mathrm{ii}}$ & $165.86(10)$ & $\mathrm{O}^{\mathrm{iii}-\mathrm{Fe} 2-\mathrm{O} 3^{\text {viii }}}$ & $103.18(9)$ \\
\hline $\mathrm{O} 1^{\mathrm{v}}-\mathrm{Fe} 1-\mathrm{O} 2^{\mathrm{i}}$ & $87.80(12)$ & 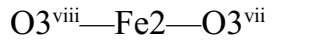 & $103.18(9)$ \\
\hline $\mathrm{O} 1-\mathrm{Fe} 1-\mathrm{O} 2^{\mathrm{ii}}$ & $87.80(12)$ & $\mathrm{O} 3-\mathrm{P} 1-\mathrm{O} 2$ & $110.28(17)$ \\
\hline $\mathrm{O} 1-\mathrm{Fe} 1-\mathrm{O} 2^{\mathrm{i}}$ & $165.86(10)$ & $\mathrm{O} 1-\mathrm{P} 1-\mathrm{O} 2$ & $111.96(17)$ \\
\hline $\mathrm{O} 1^{\mathrm{v}}-\mathrm{Fe} 1-\mathrm{O} 3^{\mathrm{iv}}$ & $89.46(11)$ & $\mathrm{O} 1-\mathrm{P} 1-\mathrm{O} 3$ & $114.29(17)$ \\
\hline $\mathrm{O} 1-\mathrm{Fe} 1-\mathrm{O} 3^{\mathrm{iii}}$ & $89.46(11)$ & $\mathrm{Fe} 1^{\mathrm{vi}}-\mathrm{O} 2-\mathrm{Fe} 2$ & $103.34(12)$ \\
\hline $\mathrm{O} 1-\mathrm{Fe} 1-\mathrm{O}^{3 \mathrm{iv}}$ & $91.67(11)$ & $\mathrm{P} 1-\mathrm{O} 2-\mathrm{Fe}^{\mathrm{vi}}$ & $127.30(17)$ \\
\hline $\mathrm{O} 1^{\mathrm{v}}-\mathrm{Fe} 1-\mathrm{O} 3^{\mathrm{iii}}$ & $91.67(11)$ & $\mathrm{P} 1-\mathrm{O} 2-\mathrm{Fe} 2$ & $128.71(18)$ \\
\hline $\mathrm{O} 1^{\mathrm{v}}-\mathrm{Fe} 1-\mathrm{O} 1$ & $99.93(18)$ & $\mathrm{Fe} 2^{\mathrm{x}}-\mathrm{O} 3-\mathrm{Fe} 1^{\mathrm{ix}}$ & $99.01(10)$ \\
\hline $\mathrm{O} 2^{\mathrm{ii}}-\mathrm{Fe} 2-\mathrm{O} 2^{\mathrm{vi}}$ & $86.57(11)$ & $\mathrm{P} 1-\mathrm{O} 3-\mathrm{Fe} 1^{\mathrm{ix}}$ & $124.27(18)$ \\
\hline $\mathrm{O} 2-\mathrm{Fe} 2-\mathrm{O} 2^{\mathrm{vi}}$ & $86.57(11)$ & $\mathrm{P} 1-\mathrm{O} 3-\mathrm{Fe} 2^{\mathrm{x}}$ & $134.56(18)$ \\
\hline $\mathrm{O} 2-\mathrm{Fe} 2-\mathrm{O} 2^{\mathrm{ii}}$ & $86.57(11)$ & $\mathrm{P} 1-\mathrm{O} 1-\mathrm{Fe} 1$ & $133.9(2)$ \\
\hline $\mathrm{O} 2-\mathrm{Fe} 2-\mathrm{O} 3^{\mathrm{iii}}$ & $92.54(11)$ & $\mathrm{O} 1-\mathrm{P} 1-\mathrm{H} 1 \mathrm{P}$ & $106.9(14)$ \\
\hline $\mathrm{O} 2^{\mathrm{vi}}-\mathrm{Fe} 2-\mathrm{O} 3^{\mathrm{viii}}$ & $92.54(11)$ & $\mathrm{O} 2-\mathrm{P} 1-\mathrm{H} 1 \mathrm{P}$ & $107.0(12)$ \\
\hline $\mathrm{O} 2^{\mathrm{ii}}-\mathrm{Fe} 2-\mathrm{O} 3^{\mathrm{vii}}$ & $92.54(11)$ & $\mathrm{O} 3-\mathrm{P} 1-\mathrm{H} 1 \mathrm{P}$ & $105.9(12)$ \\
\hline 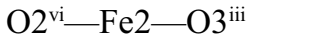 & $163.65(12)$ & & \\
\hline $\mathrm{O} 2-\mathrm{P} 1-\mathrm{O} 3-\mathrm{Fe}^{\mathrm{ix}}$ & $120.4(2)$ & $\mathrm{O} 3-\mathrm{P} 1-\mathrm{O} 1-\mathrm{Fe} 1$ & $-91.5(3)$ \\
\hline $\mathrm{O} 2-\mathrm{P} 1-\mathrm{O} 3-\mathrm{Fe}^{\mathrm{x}}$ & $-39.2(3)$ & $\mathrm{O} 1-\mathrm{P} 1-\mathrm{O} 2-\mathrm{Fe}^{\mathrm{vi}}$ & $141.7(2)$ \\
\hline $\mathrm{O} 2-\mathrm{P} 1-\mathrm{O} 1-\mathrm{Fe} 1$ & $34.8(3)$ & $\mathrm{O} 1-\mathrm{P} 1-\mathrm{O} 2-\mathrm{Fe} 2$ & $-27.5(3)$ \\
\hline $\mathrm{O} 3-\mathrm{P} 1-\mathrm{O} 2-\mathrm{Fe}^{\mathrm{vi}}$ & $-89.9(2)$ & $\mathrm{O} 1-\mathrm{P} 1-\mathrm{O} 3-\mathrm{Fe}^{\mathrm{ix}}$ & $-112.4(2)$ \\
\hline $\mathrm{O} 3-\mathrm{P} 1-\mathrm{O} 2-\mathrm{Fe} 2$ & $101.0(2)$ & $\mathrm{O} 1-\mathrm{P} 1-\mathrm{O} 3-\mathrm{Fe}^{2}$ & $88.0(3)$ \\
\hline
\end{tabular}

Symmetry codes: (i) $y, x-1,-z+1 / 2$; (ii) $-x+y+1,-x+1, z$; (iii) $-y+1,-x+1, z+1 / 2$; (iv) $x-y, x-1,-z$; (v) $x-y,-y,-z+1 / 2$; (vi) $-y+1, x-y, z$; (vii) $-x+y+1, y$, $z+1 / 2$; (viii) $x, x-y, z+1 / 2$; (ix) $y+1,-x+y+1,-z$; (x) $-y+1,-x+1, z-1 / 2$. 